\title{
The Verificatioin of 『Chinese Teaching Method』Class Effectiveness by Observational Evaluation
}

\author{
『중국어 교수법』관찰평가를 통한 수업효과 검증 \\ In-Suk Jung ${ }^{1}$, Soo-Koung Jun ${ }^{2}$ \\ 정인숙 ${ }^{1}$, 전수경 2 \\ ${ }^{1}$ Professor, Department of China, Namseoul University, South Korea,woxingfu@nsu.ac.kr \\ ${ }^{2}$ Professor, College of General Education, Namseoul University, South Korea, \\ skjun74@nsu.ac.kr
}

Corresponding author: Soo-Koung Jun

\begin{abstract}
This study aimed to verify the effectiveness of the "Chinese Teaching Method" class through observational evaluation of pre and post changes in the core competencies. Two observational evaluation isomorphic checklists (types A and B) developed by the university were used to measure core competencies. At the beginning and end of the classes, students were also evaluated of their satisfaction throughout the course. This class was one of the teaching subjects, and because there were a small number of 5 participants, it was possible to observe and evaluate the students' competencies (communication \& creativity). The scores of the observed checklist were evaluated using a 5-point Likert scale, the mean and standard deviation were calculated, and the change of the score before and after was verified by Wilcoxon nonparametric statistics. In both communication and creative competency, the post-test scores were significantly improved than the pre-test, and the lecture evaluation by students was also higher overall, which proved that the lessons were effective in promoting communication and creative competencies. Through these results, it is meaningful to present an example that can more clearly practice competency-based instruction in class for a small number of students and verify the effect through observational evaluation. In the future, it is expected that it will be able to give implications for designing specific and clear classes that can cultivate students' competencies.
\end{abstract}

Keywords: Chinese Teaching Method, Core Competency, Observational Evaluation, Class Effectiveness

요약: 본 연구 목적은 「중국어 교수법」 수업의 효과성을 핵심역량의 사전-사후 변화를 관찰 평가하여 검증하고자 하는 것이다. 핵심역량의 측정 도구는 대학에서 개발된 관찰평가 동형검사지 $\mathrm{A}, \mathrm{B}$ 형) 두 가지를 학기 초와학기 말 수업이 종료되는 시점에 실시하였다. 또한 학생들에게 강의 평가를 실시하여 강의 전반에 걸쳐 만족도를 살펴보았다. 본 수업은 교직과목 중의 하나로 참여자가 5 명으로 소수이기 때문에 역량 관찰평가가 가능했으며, 주 역량은 소통이고, 부역량은 창의역량이다. 관찰된 체크리스트의 점수는 5점 리커리트로 평가되었고 평균과 표준편차를 산출하였으며, 사전과 사후의 점수 변화를 Wilcoxon 비모수 통계로 검증하였다. 소통과 창의역량 모두

Received: June 06, 2021; $1^{\text {st }}$ Review Result: July 23, 2021; $2^{\text {nd }}$ Review Result: September 08, 2021 Accepted: October 31, 2021 
사전검사보다 사후검사 점수가 의미 있게 향상되었으며, 강의평가도 전반적으로 높게 나타나, 소통과 창의역량을 증진하는데 수업이 효과적인 것으로 검증되었다. 이러한 결과를 통해 소수를 대상으로 하는 수업에서는 좀 더 명확하게 역량기반 중심 수업을 실천하고 그 효과를 관찰평가를 통해 검증할 수 있는 사례를 제시한 것에 의미가 있다. 앞으로도 학생들의 역량을 배양할 수 있는 구체적이고 명확한 수업을 설계하는 데 시사점을 줄 수 있을 것으로 기대된다.

핵심어: 중국어 교수법, 핵심역량, 관찰평가, 수업효과성

\section{1. 서론}

과거 어느 때 보다 지금의 대학교육에서는 21 세기 지식기반 사회에서 중시되던 지식습득을 중심으로 하는 교육이 아니라 역량을 갖춘 인재를 양성하는 것이 중요한 과제가 되고 있다. $\mathrm{OECD}$ 는 1997년 DeSeCo(Definition and Selection of Key Competences) 프로젝트에서 글로벌화되어 가고 있는 지금의 시대에서 삶의 질을 높이기 위한 역량을 제안한 바 있다. 'DeSeCo'는 미래 사회에서 개인이 반드시 갖춰야 하는 3 대 핵심 역량 범주를 가리키는 말로써, '도구의 지적활용(Use tools interactively)', '사회적 상호작용(Interact in heterogeneous groups)' , ‘자율적 행동(Act autonomously)' 등이 포함된다[1]. 우리나라의 교육부에서는 대학교육을 중심으로 한 국책사업에서도 역량기반(competency-based) 교육의 중요성을 계속 강조해 오고 있다. ACE(Advancement of College Education, 학부 교육 선진화 선도대학육성사업), LINC(Leaders in Industryuniversity Cooperation, 산학협력선도대학육성사업), CK(University for Creative Korea, 대학특성화사업) 등 국책사업에서도 역량기반 교육이 중요한 평가 요소로 설정되어 있다.

충남에 위치한 $\mathrm{N}$ 대학에서도 역량을 기반으로 교육과정을 편성하고 운영하고 있다. 역량기반 교육과정은 교양 교육과정 개편과 전공 교육과정 개편의 기본적인 개념과 원리로 설정하고, 교육과정을 이수하는 학생들에게 능력과 역량을 배양하고자 한다. 특별히 대학의 핵심가치와 교육목표, 인재상을 반영한 핵심역량 5개를 설정하고 개설되는 교과목과 매칭하고 있다. 5개 핵심역량은 소통(communication), 협업(collaboration), 융합(convergence), 창의(creativity), 그리고 글로컬 (glocal)이다. 각 교과목 수준에서 수업의 목표를 설정할 때는 교과목에서 주 역량과 부역량으로 설정하고 있는 역량의 요소가 반영되어 기술되도록 하고 있다. 따라서 특정 교과목 수업을 이수한다고 할 때는 수업에서 설정된 역량이 수업을 듣기 전과 듣고 난 후에 변화를 통해 수업의 효과성을 검증할 수 있다.

본 대학에서는 핵심역량을 진단할 수 있는 도구로서 학생들의 자가진단 검사뿐만 아니라 교수자가 수업에서 나타내는 학생의 수행(performance)을 관찰하면서 체크할 수 있는 '관찰형 검사' 를 개발하였다. 이러한 핵심역량 관찰형 검사는 교과 및 비교과 효과성 검증에 활용될 수 있다. 본 연구의 목적은 관찰형 검사 도구를 활용한 역량의 사전-사후 검사를 통해 수업의 효과성을 검증하고자 한다. 이러한 연구목적을 달성하고자 설정된 구체적인 연구 문제는 『중국어 교수법』 수업을 통해 학생들의 핵심역량에는 어떠한 변화가 있는가? 로 설정하였다. 


\section{2. 이론적 배경}

\section{1 역량과 핵심역량}

선행연구에 따르면 역량은 특정 분야에서 가장 효율적으로 역할을 수행하면서 성과를 창출하도록 하는 행동과 기술, 지식이라고 볼 수 있다[2]. White(1959)는 역량의 개념을 '환경과 효과적으로 상호작용하는 능력' 으로 소개하였다[3]. McClelland(1973)는 '특정 조직이나 환경에서 필요로 하는 것을 이룰 수 있는 능력' 으로 역량을 정의하였다[4]. Spencer와 Spencer(1993)는 특정한 상황이나 직무에서 준거에 따른 효과적이고 우수한 수행의 원인이 되는 개인의 내적인 특성으로 역량을 정의하였으며, McLagan(1989)은 특정 영역에서 결과물을 산출해 내는 데 결정적인 영향을 주는 지식과 기술이라고 정의하였다[5][6]. 1997년부터 2003년까지 진행된 OECD DeSeCo(Definition and Selection of Key Competencies) 프로젝트에서는 역량을 지식과 기술뿐만 아니라 개인의 태도, 가치, 동기와 같은 심리.사회적 자원을 이용하여 특정 맥락의 복잡한 요구에 대처하는 능력으로 정의하고 있다[7]. $\mathrm{OECD}$ 는 삶에 필요한 수많은 역량 중 경제적, 사회적 목적에 부합하는 혜택을 제공하며, 삶의 다양한 맥락에 적용되며, 모든 사람에게 필요한 역량을 ‘핵심역량(key competencies)' 으로 규정하였다.

\section{2 대학의 핵심역량기반 수업}

오랫동안 대학교육의 교육과정은 학문 중심교육과정이다. 전통적인 유형이지만 시대와 변화에 따라서 다양한 형태로 나타나고 있는데, 그중의 하나가 역량기반 교육과정이다. 역량기반 교육과정은 현대 사회의 성공적 삶을 위한 총체적 능력으로서의 역량 개념을 설정하고 대학 교육과정에 적용하는 것을 의미한다[8].

우리나라의 많은 대학에서는 핵심역량을 설정하고 역량을 기반으로 하는 교육과정과 수업 설계를 하고 있다. 대학교육에서 역량기반 교육의 필요성이 강조됨에 따라 $\mathrm{ACE}$, $\mathrm{LINK}, \mathrm{CK}$ 등 사업에 참여하는 대학을 중심으로 역량기반의 교육이 도입되어 왔다. 특히 $\mathrm{ACE}$ 사업에 참여하는 대학교의 경우 학교 차원의 핵심역량 목표를 개발하고 역량기반의 전공/교양/비교과 교육과정을 개발 및 운영하도록 하였다. 또한 이들 대학에서는 학생의 핵심역량 수준을 진단하기 위해 자체적인 진단도구를 개발하거나 K-CESA(Korea Collegiate Essential Skils Assesment)의 조사에 참여하고 있다[9].

충남에 소재한 N대학에서는 2013년부터 5대 핵심역량(소통, 섬김, 창의, 글로벌, 실무역량)을 개발하고, 교양교육과목을 중심으로 핵심역량 설정하고 수업계획서에 역량을 반영하도록 한 후 점차 전공교과목에 역량 설정으로 확대해 나갔다. 2018년에는 대학의 중장기 발전계획 등에 따른 핵심역량 재설정(소통, 협업, 창의, 융합, 글로컬)이 이루어졌으며, 이를 기반으로 교양교육과정을 편성하고 2019학년도부터 교육과정이 개시되었다. 전공교육과정은 2020학년도부터 핵심역량과 전공역량이 모두 적용되어 개편, 운영되고 있다.

\section{3 중국어 교수법과 핵심역량}

$\mathrm{N}$ 대학에서 『중국어 교수법』 교과목은 대학의 핵심역량 중 소통역량과 창의역량과 연관성을 설정하고 있다. 교과목과 연계된 핵심역량은 수업에서 달성하고자 하는 
수업목표로 설정되고, 수업을 통해 역량이 향상되도록 하고 있다. 먼저, 소통역량과의 관련성을 살펴보면, 『중국어 교수법』은 중국과 중국어에 대한 이해를 기본으로 하고 있기 때문에, ‘소통' 과 밀접한 관련성을 갖는다. 본 수업을 듣는 학생들은 듣기, 읽기, 쓰기, 말하기 토론 및 상황에 적절한 이해 및 전달 능력을 ㄴ, 있있어야 하기 때문이다. 또한 중국을 포함한 타문화에 대한 이해를 바탕으로 타인과 소통할 수 있어야 하기 때문이다. 둘째, 본 수업은 '창의' 역량과 관련성을 갖는다. 예비교사를 위한 수업으로써, 교사는 수업을 설계할 때, 학생들의 니즈와 흥미 등의 수준을 고려하여 교수법을 설정하고 수업을 이끌어 나갈 수 있어야 하기 때문이다. 교수법의 성격상 상황에 적절한 교수법을 개발하고 적용하며, 수업 중에 발생할 수 있는 문제 등을 창의적으로 해결할 수 있어야 하기 때문이다. 즉, 다양한 학생들의 눈높이에 맞춰 교육을 할 수 있는 것과 교육과 수업에서 발생하는 문제를 해결하는 역량을 갖추는 것이다.

본 『중국어 교수법』 수업에서는 소통역량 증진을 위해서 중국어 의사소통의 요소를 이루는 말하기, 듣기, 쓰기, 읽기 및 문법, 어휘, 그리고 더 나아가 문화적인 소통이 이루어질 수 있는 지도와 실습을 하게 된다. 그리고 창의역량 개발을 위해서 창의 교수법 교안 개발과 창의적인 교수법을 적용한 수업 시연과 동료 및 교수자 피드백을 통해 수업 역량을 개발해 나간다.

\section{4 관찰(수행)평가와 교육효과성}

$\mathrm{N}$ 대학에서는 핵심역량을 진단하는 도구를 개발하고 학생들의 자가진단 역량 검사를 매년 실시하며 추이를 분석하고, 교육과정 개편과 수업 개선에 활용하고 있다. 역량을 측정한다는 것은 실제 학생이 그러한 능력을 갖추고 있는지를 측정해야 하기 때문에 지필 평가보다는 수행을 관찰하는 평가가 더욱 적합할 것이다. 그러나 소수의 인원을 대상으로 하는 실험 · 실습·실기 수업이 아닌 경우 많은 인원의 학생들을 대상으로 하는 이론 중심의 수업에서는 학생들의 역량을 평가하기는 쉽지 않다. 따라서 대부분의 핵심역량 측정을 위한 진단 도구는 학생들 자신의 자가진단 평가를 기반으로 하고 있다.

$\mathrm{N}$ 대학에서는 2018년 자가진단 평가의 한계를 보완하고자 핵심역량 관찰평가 도구를 개발하였다. 관찰평가는 교수자가 학생을 관찰하면서 정보를 얻는 평가 방법을 의미한다. N 대학에서는 5 대 핵심역량별로 9 개씩의 관찰 문항을 제작하였고, 각 문항은 3 개의 수준으로 체크가 되도록 구성되어 있다. 그리고 역량별 관찰평가 검사는 2 종으로 개발되어 사전-사후 검사에서 각각 사용할 수 있게 되어 있다. 관찰평가는 평가자가 학생들의 수행 수준을 평가하는 것으로 수행평가로 이해할 수 있다. 수행평가(performance)는 실제로 행동을 하는 과정이나 행동의 결과를 관찰해서 판단하는 평가이다[10]. 수행의 정도를 관찰로 평가하여 역량의 증감을 확인해서 교육의 효과성을 검증하는 데 활용할 수 있다.

교육의 효과성(effectiveness)이란 교육을 통해 습득한 지식, 기술, 태도 등을 업무 상황에서 효과적으로 적용하는 것을 의미한다고 보았으며(Tracey et al., 1995), Kirkkpartick(1994)은 교육 효과를 반응, 학습, 행동, 결과로 구분하여 교육의 효과가 순차적, 인과적, 계층적으로 발생한다고 하였다[11][12]. 교육의 효과를 측정하는 한 가지 방법은 프로그램이나 교육 이수 전에 측정한 결과와 교육 이수 후에 측정한 결과의 차이를 보는 것이며, 본 연구에서는 사전관찰과 사후관찰 평가의 차이를 통해 교육의 
효과성을 검증하는 방법을 채택하였다.

\section{3. 연구방법}

\section{1 연구대상}

본 연구는 핵심역량의 증감을 바탕으로 중국어 교수법 수업의 효과성을 탐색하고자 2020년 2학기 중국어 교수법 수업에 참여한 학생 5명을 대상으로 관찰평가를 하였다. 중국어 교수법은 예비교사 양성과정의 교과교육과목의 하나로 교직이수 신청자에 한해서 수업이 개설되고 있기 때문에 5명의 학생은 모두 교직이수 대상 학생이다. 5명 모두 여학생이며, 3학년이 3명(60\%), 4학년이 2명(40\%)이다.

[표 1] 연구대상

[Table 1] Research Subjects

\begin{tabular}{c|c|c|c}
\hline 구분 & 세부구분 & 명 & $\%$ \\
\hline 성별 & 여 & 5 & 100.0 \\
\hline \multirow{2}{*}{ 학년 } & 3 학년 & 3 & 60.0 \\
& 4 학년 & 2 & 40.0 \\
\hline
\end{tabular}

\section{2 연구 방법과 절차}

본 연구는 2020년 11월에 관찰평가를 통해서 학생들의 핵심역량을 측정했으며, 5주의 수업이 끝난 뒤 12 월 사후검사로 관찰평가를 재-실시하였다. 수업관찰을 위해서 만들어진 핵심역량 관찰평가용 체크리스트를 가지고 사전-사후 평가를 하였다. 수업관찰은 관찰자 2 명이 하고 평균을 내었으며, 사전과 사후의 증감률을 계산하였다. 관찰자 1 은 핵심역량 전문가로서 대학의 성과관리센터 소속의 교수자이며, 관찰자 2 는 중국어 교수법 수업을 담당하는 중국어 전공 교수자이면서 핵심역량 관찰평가를 위한 훈련을 이수하였다.

\section{3 연구 측정도구 및 분석}

본 연구를 위해서 사용된 핵심역량 관찰평가 체크리스트는 대학의 교육성과관리센터에서 개발한 관찰평가용 검사지로서 동형 검사지이다. 사전검사에서는 $\mathrm{A}$ 형 검사지를 사용하였으며, 사후검사에서는 B형 검사지를 사용하였다. 각 역량마다 9문항으로 구성되어 있어서 본 연구에서는 소통역량 9문항, 창의역량 9문항으로 총 18 문항을 사용하였다. 각 문항은 3 개의 수준(상, 중, 하)로 구성되며, 관찰에 따라서 수준을 체크하는 방식으로 이루어진다(표 2). 사전-사후의 검사 구성은 <표 $3>$ 과 같다. 또한 강의가 종료된 후 강의평가를 실시하였으며, 강의평가는 대학에서 제작된 평가지로서 모든 수업에 적용되는 평가지로서, 총 9개 영역으로 구성된다(표 5). 본 연구는 조사 수집된 자료의 코딩은 마이크로소프트 엑셀(Microsoft Excel)을 사용하여, 평균과 표준편차를 산출하였다. 
[표 2] 관찰평가 체크리스트 문항 사례

[Table 2] Examples of Observational Evaluation Checklist Item

\begin{tabular}{|c|c|c|}
\hline 역량 & 행동사례(수준별) & 수준 \\
\hline \multirow{3}{*}{ 소통역량 } & 학생은 상대방이 말하는 의미를 이해한다. & 하 \\
\hline & 학생은 상대방이 말하는 참된 의미를 제시 할 수 있다. & 중 \\
\hline & 학생은 상대방이 말하는 참된 의미를 분석할 수 있다. & 상 \\
\hline \multirow{3}{*}{ 창의역량 } & 학생은 위기상황에 따른 극복의 중요성을 안다. & 하 \\
\hline & 학생은 위기상황에 따른 극복방법을 적용할 수 있다. & 중 \\
\hline & 학생은 위기상황에 따른 극복결과를 평가할 수 있다. & 상 \\
\hline
\end{tabular}

[표 3] 사전-사후 검사 구성

[Table 3] Pre and Post Test Composition

\begin{tabular}{|c|c|c|c|}
\hline (사전, 후) 동형검사 & 사전검사(가형) & 사후검사(나형) & 문항수 \\
\hline 소통역량 & $1 \sim 9$ & $1 \sim 9$ & 9 개 문항 \\
\hline 창의역량 & $1 \sim 9$ & $1 \sim 9$ & 9개 문항 \\
\hline
\end{tabular}

\section{4. 연구결과}

\section{1 핵심역량의 사전-사후 관찰평가 차이}

본 연구에서는 중국어 교수법 수업에서 설정한 핵심역량의 사전-사후검사 점수의 증감을 통해서 수업의 효과성을 검증하고자 하였다. 주역량인 소통역량의 경우, 사전검사는 평균 $1.57(\mathrm{SD}=.32)$ 이고, 사후검사의 평균은 $2.93(\mathrm{SD}=.15)$ 로 1.37 이 증가하였고, 수업은 소통역량 향상에 효과가 있는 것으로 보인다 (증가률 $87.2 \%$ ). 부역량으로 설정한 창의역량도 사전검사는 평균 $1.41(\mathrm{SD}=.34)$, 사후검사 평균 2.91( $\mathrm{SD}=.20)$ 로 효과가 있는 것으로 나타났다(증가율 106.3\%). 비모수 통계로 사전-사후 검사 차이 대응표본 검증을 한 결과, 소통역량은 0.043 , 창의역량은 0.042 로 유의미한 차이가 있는 것으로 나타났다. 결과는 [표 4]와 같다.

[표 4] 핵심역량 사전-사후 검사결과

[Table 4] Core Competency Pre-Post Test Results

\begin{tabular}{|c|c|c|c|c|c|c|}
\hline 역량 & 학생 & 사전평균(SD) & 사후평균(SD) & 증감 & 증감률(\%) & Wil coxon \\
\hline \multirow{5}{*}{ 소통역량 } & 1 & 1.78 & 3.00 & $\mathbf{\Delta} 1.22$ & \multirow{5}{*}{87.2} & \multirow{5}{*}{.043} \\
\hline & 2 & 1.28 & 2.67 & $\boldsymbol{\Delta} 1.39$ & & \\
\hline & 3 & 2.00 & 3.00 & $\Delta 1.00$ & & \\
\hline & 4 & 1.28 & 3.00 & $\boldsymbol{\Delta} 1.72$ & & \\
\hline & 5 & 1.50 & 3.00 & $\Delta 1.50$ & & \\
\hline
\end{tabular}




\begin{tabular}{|c|c|c|c|c|c|c|}
\hline & 전체 & $1.57(\mathrm{SD}=.32)$ & $2.93(\mathrm{SD}=.15)$ & $\Delta 1.37$ & & \\
\hline \multirow{6}{*}{ 창의역량 } & 1 & 1.72 & 3.00 & $\Delta 1.28$ & \multirow{6}{*}{106.3} & \multirow{6}{*}{.042} \\
\hline & 2 & 1.50 & 3.00 & $\Delta 1.50$ & & \\
\hline & 3 & 1.72 & 3.00 & $\boldsymbol{\Delta} 1.28$ & & \\
\hline & 4 & 1.11 & 2.56 & $\boldsymbol{\Delta} 1.44$ & & \\
\hline & 5 & 1.00 & 3.00 & $\boldsymbol{\Delta} 2.00$ & & \\
\hline & 전체 & $1.41(\mathrm{SD}=.34)$ & $2.91(\mathrm{SD}=.20)$ & $\Delta 1.50$ & & \\
\hline
\end{tabular}

\section{2 학생들의 강의평가를 통해서 본 수업효과성}

수업이 종료된 후 학생들에게 강의평가를 무기명으로 실시하였다. 강의평가는 학생들이 수업과 교수자에 대한 평가를 의미하는 것으로, 수업만족도, 수업계획, 교수방법, 진행속도, 수업준비, 성적평가, 수업환경, 학습역량으로 총 9개 영역에서 5점 리커르트 척도로 이루어진다. 구체적인 결과는 <표 5>와 같다. '수업에 대한 전반적인 만족' '교수-학습방법에 대한 전반적인 만족도' , '객관적인 성적평가' ,

'학습역량을 키워주는 수업' 에 대해서 5점 만점을 받았으며, '교수자의 수업준비의 충실성' 이 4.9, ‘수업계획서에 따른 충실한 수업진행' 이 4.8, '학생의 능력과 이해수준을 고려한 적절한 진행 속도' 가 4.6, 그리고 '수업환경' 이 4.5의 순서로 나타났다.

[표 5] 학생들이 실시한 강의평가 결과

[Table 5] Lecture Evaluation Results by Students

\begin{tabular}{l|l|l|c}
\hline 번호 & 영역 & 내용 & 평점 \\
\hline 1 & 수업만족 & 전반적인 수업만족도 & 5 \\
\hline 2 & 수업계획 & 수업계획서에 따른 충실한 수업진행 & 4.8 \\
\hline 3 & 교수방법 & 교수-학습방법에 대한 만족도 & 5 \\
\hline 4 & 진행속도 & 학생의 능력과 수준을 고려한 진행속도 & 4.6 \\
\hline 5 & 수업준비 & 교수자의 수업준비도 & 4.9 \\
\hline 6 & 성적평가 & 객관적인 성적평가 & 5 \\
\hline 7 & 수업환경 & 효과적인 수업지원이 이루어지는 수업환경 & 4.5 \\
\hline 8 & 학습역량 & 학습역량을 높여주는 수업 & 5 \\
\hline
\end{tabular}

\section{3 관찰을 통한 학생의 역량 정성평가}

사전관찰과 사후관찰 평가를 통해서 소통역량과 창의역량에 대한 교수자의 학생별 정성적 평가 내용은 다음과 같다.

\subsection{1 소통역량의 사전-사후평가}

$\mathrm{a}$ 학생 : [사전] 학습자들의 눈높이에서 소통을 하고자 노력 하며, 질문으로 밝은 수업 분위기를 만들려고 하나 아직 경험부족으로 본인이 준비한 수업을 설명하는 데에 급급함이 보임. $\rightarrow$ [사후] 사전시연에서는 긴장과 자신감이 부족하여 학습자들과의 
소통이 약간 부족해 보였는데 사전시연 후 동료피드백과 교수피드백을 참고로 하여 연습한 결과 소통역량이 개선 되었다.

$\mathrm{b}$ 학생 : [사전] 학습자에게 질문을 하면서 상대방의 의중은 파악하나 그에 따른 적절한 반응을 하는 것에서는 약간 미숙하지만 관심을 가지며 해결하려 하는 노력의 자세는 돋보였다. $\rightarrow$ [사후] 학습자의 질문에 대한 대답을 할 때 확실히 사전평가 때 보다는 여유가 생겼다. 모르는 것에 대해서는 모른다고 애기하는 여유와 순간 임기응변으로 대처하는 능력도 나아졌다.

c학생 : [사전] 수업을 할 때 소통의 문제는 없으나 약간 수업 진행이 빠르다는 느낌을 받았다. 학습자의 적절한 반응과 표정을 읽으면서 여유롭게 수업을 진행한다면 좋은 수업이 될 것 같다. $\rightarrow$ [사후] 학습자의 수준을 고려해서 쓰기교육을 문장을 활용하면서 하는 수업이 좋았다. 사전평가에서 보다 여유와 재치가 돋보여 한결 수업이 편안하게 느껴졌다.

$\mathrm{d}$ 학생 : [사전] 학습자의 수준을 고려해서 수업을 하기 보다는 본인이 준비해 온 수업의 내용을 설명하기에 급급해 하는 모습이여서 좀 아쉬었다. 많은 것을 학습자에게 알려주려고만 하는 느낌이 들었다. 개선해야 할 부분인거 같다. $\rightarrow$ [사후] 사전평가시 지적되었던 일방적인 설명식 소통식의 수업이 많이 개선 되었다. 학습자들과 아이컨텍을 하면서 수업을 이끄는 여유로운 자세도 보기 좋았다.

e학생 : [사전] 소통을 하려는 자세는 좋았지만 쑥스러움을 많이 타는 성격이라 소심함이 소통하는 데에 장애로 작용하고 있다. 또한 목소리 연출도 조금 신경 써야 할 것 같다. $\rightarrow$ [사후] 학습자의 반응에 자신있게 대처하려는 자심감이 향상되었고, 학습자의 감정에 적절히 표현하려는 여유로운 자세도 보였고, 사전평가보다는 소통역량이 많이 나아졌다

\subsection{2 창의역량의 사전-사후평가}

$\mathrm{a}$ 학생 : [사전] 다양한 콘텐츠를 사용하여 학습자들의 호기심을 유발하고, 질문을 통해 학습자들의 창의역량을 배양하려 노력하는 모습이 보이긴 하지만 본인 스스로 긴장을 하고 있어 창의적 역량을 크게 발휘 하지 못함. $\rightarrow$ [사후] 돋보이는 콘텐츠와 재매있는 활동은 학습자로 하여금 창의성을 길러주기에 충분했다고 할 수 있다. 그러나 한자 육서부분을 가르칠 때에는 일반적인 설명식이 주가 되어 학습자들로 하여금 약간의 지루함을 느낄 수도 있겠다는 생각이 들었다.

$\mathrm{b}$ 학생 : [사전] 학생의 질문에 적절히 반응하나 자신감 있는 대처는 아니었다. 그러나 학습지도안의 교수의 콘텐츠활용과 활동은 창의적 이었다. $\rightarrow$ [사후] 학습자의 눈높이에서 기본기부터 탄탄히 자세히 가르치려는 자세가 좋았고, 중국어 문장의 예를 들어 설명하는 부분이 창의적이어 인상적이었다.

c학생 : [사전] 탄탄한 논리로 학습자들로 하여금 이해가 쉽게 갈 수 있도록 설명하는 부분이 참 좋았다. 수업콘텐츠를 좀 더 창의적인 것을 사용하면 좋을 것 같다는 생각이 들었다. $\rightarrow$ [사후] 추상적인 내용을 창의적 콘텐츠와 연결지어 설명하는 모습이 굿 아이디어라 생각한다. 그리고 문법적 설명도 창의적으로 설명하는 것이 인상 깊었다.

$\mathrm{d}$ 학생 : [사전] 학많은 양을 학습자들에게 알려주려 하다 보니 수업이 단조로움을 느끼게 하였다. 좀 더 여유를 갖고 창의적콘텐츠와 창의적 교수법을 활용하면 좋을 것 같다. $\rightarrow$ [사후] 그림의 콘텐츠를 가지고 쓰기교육의 접근은 창의적이라 할 수 있다. 또한 그림을 가지고 교수자가 먼저 다양한 쓰기의 예를 들어주는 자세도 보기 좋았다. 
e학생 : [사전] 중국어 어법 기본에 튼튼한 수업구성이며 콘텐츠 또한 창의적인 면이 있지만 자신감 부족으로 창의력을 맘껏 발휘하지 못하는 것이 아쉬움으로 남는다. $\rightarrow$ [사후] 자신감의 향상이 창의력의 발휘에도 미치는 것 같다. 창의적 콘텐츠 할용을 사전평가에서는 미흡했는데 사후평가에서는 많이 향상된 것을 볼 수 있었다.

\section{5. 결론 및 논의}

본 수업은 미래의 중국어 교사의 기본 자질을 갖추기 위한 교직 과목으로서 중국어 교수법에 관한 이론과 교수 기술을 중점적으로 배운다. 4주 차까지는 외국어로서의 중국어 교수법에 관한 이론들을 다룬 뒤, 5 주 차부터는발음지도에 관한 이론을 배우고 수업 시연과 동료 및 교수 피드백을 받고, 어휘지도와 수업 시연(피드백), 어법 지도 및

수업 시연(피드백), 듣기지도 및 수업 시연(피드백), 말하기 지도 및 수업 시연(피드백), 문화지도 및 수업 시연(피드백), 쓰기 지도 및 수업 시연(피드백) 등으로 수업 시연을 하면서 실습과 실무경험을 하게 된다.

이를 위해 먼저 실현 가능한 수업지도안 작성을 해야 하며, 수업 지도안을 토대로 수업 시연을 하면서 창의적 교수법과 의사소통 능력을 갖추어야 한다. 이러한 목적을 위해서는 수업 지도안 내용이 창의적으로 구성되어야 하며, 수업 시연에서 주-역량인 소통역량을 강화할 수 있는 수업으로 진행되어야 한다. 창의역량과 소통역량이 융합되어 학습자의 눈높이에서 수업을 진행하면서 학습자로 하여금 중국어에 대한 흥미 유발이 일어날 수 있는 수업을 하는 것이 중요하다고 할 수 있다.

본 연구는 이러한 중국어 교수법 수업을 핵심역량을 기반으로 사전-사후 관찰평가를 실시함으로써 수업의 효과성을 검증하고자 하였다. 먼저, 주 역량인 소통과 부역량의 창의역량에서 사전검사보다 사후검사에서 모두 점수가 향상됨으로써 수업의 효과성을 보여주었다. 둘째, 학생들의 강의 평가를 통해서 수업 효과성을 살펴보았을 때에도 9 개 영역 모두에서 높은 점수를 나타냈고 만족도 수준이 높게 나타났다. 전반적인 만족도가 높은 것은 학생들의 체험 위주와 학생들의 눈높이에서 수업한 결과라고 본다. 교수 방법에 대한 만족도는 의사소통식 교육을 한 결과이고 학생들이 모의 수업 시연을 한 후 동료피드백과 교수피드백의 효과라고 할 수 있다. 수업의 진행속도는 학생들의 수준에서 토론과 토의의 수업으로 진행하면서 서로의 다름을 인정하며 배려하는 분위기 속에서 진행하였다. 수업 준비는 시연이 대부분인 교과목의 특성상 먼저 학생들의 자신감과 자존감을 높일 수 있는 수업을 위해 수업준비를 실시한 결과 학생들이 만족하는 평가를 한 것이라 볼 수 있다. 성적평가에서는 학생들이 자기 자신을 평가한 것과 동료피드백 및 교수피드백을 참고하여 평가하였다. 이러한 평가가 모의시연을 객관적으로 평가할 수 있는 방법이라 여겨지며 또한 학생들에게도 만족하는 평가가 되었다. 모든 영역에서 의사소통을 중시하며 학생들의 눈높이 수준에서 수업한 결과 본 수업의 학생들 역량향상에도 긍정적인 효과를 가져왔다고 할 수 있다. 마지막으로 학생들에 대한 교수자의 사전-사후 관찰 노트를 통해서 학생 개개인별로 소통역량과 창의역량이 향상된 영역을 확인할 수 있었다.

중국어 교수법을 숙지하고 수업시연을 할 때 자신감·도전정신·용기가 부족하여 시연에서 자신이 준비한 수업내용을 제대로 발휘하지 못할 경우가 많다. 따라서 수업시연의 기회를 확대하고 피드백을 통해 학생들이 마인드 콘트롤을 하면서 수업시연을 더욱 능숙하게 잘 할 수 있도록 돕는 것이 중요할 것이다. 이를 위해서 
수업지도안 작성부터 교수자의 피드백을 강화해서 탄탄한 수업계획을 기반으로 자신감 넘치는 수업시연이 되도록 해야 할 것이다.

핵심역량에 대한 사전-사후 관찰평가는 수업에 참여한 학생들의 역량을 주도 면밀하게 평가하면서 학생들의 변화를 볼 수 있다는 점에서 큰 장점이 있으며, 교수자 입장에서도 수업을 어떻게 개선해야 할 지에 관해서 구체적인 대안을 찾을 수 있는 기회를 제공하는 장점이 있어 보인다. 예를 들어 핵심역량을 기반으로 설정된 수업 목표 중에 문제해결력이라는 부분이 미흡한 학생이 관찰되면 학생 개별적인 조언과 상담, 그리고 교안을 처음부터 재구조화 및 재작성 등을 하도록 지도할 수 있다. 하지만 본 연구는 일개 수업을 대상으로 소수의 학생을 대상으로 하였기 때문에 일반화 하기 어려운 한계점을 지닌다. 본 대학에서 다양한 수업에서 핵심역량 관찰 평가가 조금더 확대 적용되어 수업의 효과성을 측정하는 관찰평가의 타당성을 더욱 확보할 필요가 있을 것이다. 추후에는 본 연구에서 다뤄진 소통과 창의역량 이외에도 글로컬, 협업, 융합 등의 핵심역량도 관찰평가가 이루어지는 연구가 필요하다.

\section{References}

[1] S. Y. Lee, A study on the OECD DeSeCo and education 2030 project: some implications for school education, Seoul National Education University, Master's Thesis, (2019)

[2] Y. K. Kim, A study on the effectiveness of core competency courses according to facilitating strategy of learning transfer focusing $\mathrm{H}$ Corporation, Journal of Digital Convergence, (2014), Vol.12, No.8, pp.175-186, DOI: 10.14400/JDC.2014.12.8.175

[3] R. W. White, Motivation reconsidered: the concept of competence, Psychological Review, (1959), Vol.66, No.5, pp.297333, DOI: $10.1037 / \mathrm{h} 0040934$

[4] D. C. McClelland, Testing for competence rather than for intelligence, American Psychologist, (1973), Vol.29, No.1, pp.1-14, DOI: 10.1037/h0040934

[5] L. M. Spencer, S. M. Spencer, Competence at Work: models for superior performance, John Wiley \& Sons, (1993)

[6] P. A. McLagan, Models for HRD practice(4th ed.), ASTD, (1989)

[7] OECD, The definition and selection of key competences, (2005), https://www.oecd.org/pisa/35070367.pdf

[8] E. J. Kim, M. H. Sung, Analysis of changes in core competencies and class satisfaction of college students according to the core competencies-based liberal arts curriculum, Journal of the Korean Society for Core Competency Education Conference, (2018), Vol.4, pp.93-109, http://scholar.dkyobobook.co.kr/searchDetail.laf?barcode=4050026248092\#

[9] P. S. Kim, S. D. Kim, Understanding about the experience of university professors' competence-based class operation, Journal of Narrative and Educational Research, (2017), Vol.5, No.2, pp.89-115, DOI: 10.25051/jner.2017.5.2.005

[10] D. H. Kwon, Educational Evaluation, Hakjisa, (2016)

[11] J. B. Tracey, S. I. Tannenbaum, M. J. Kavanaugh, Applying trained skills on the job: the importance of the work environment, Journal of Applied Psychology, (1995), Vol.80, No.2, pp.239-252, DOI: 10.1037/0021-9010.80.2.239

[12] D. L. Kirkpatrick, Evaluating Training Program: The Four Levels, Berrett-Koehler, (1994) 